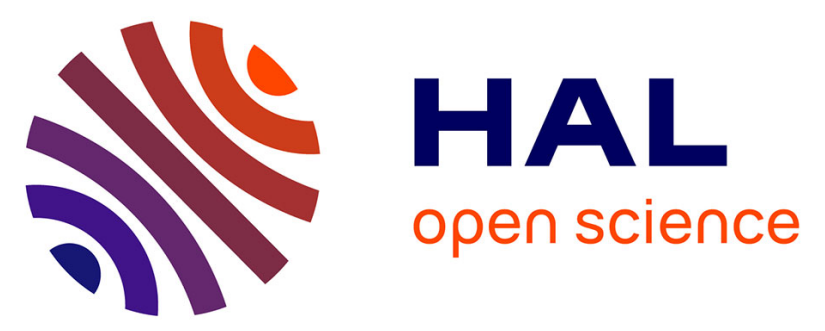

\title{
PHYSICAL PROPERTIES OF ULTRA THIN FILMS STUDIED BY X-RAY OPTICAL TECHNIQUES : LANGMUIR-BLODGETT MULTILAYER STRUCTURES, ORGANIC MONOLAYERS ON WATER AND THE SPREADING OF POLYMER MICRO-DROPLETS
}

\author{
J. Benattar, J. Daillant, Louis Bosio, L. Leger
}

\section{- To cite this version:}

J. Benattar, J. Daillant, Louis Bosio, L. Leger. PHYSICAL PROPERTIES OF ULTRA THIN FILMS STUDIED BY X-RAY OPTICAL TECHNIQUES: LANGMUIR-BLODGETT MULTILAYER STRUCTURES, ORGANIC MONOLAYERS ON WATER AND THE SPREADING OF POLYMER MICRO-DROPLETS. Journal de Physique Colloques, 1989, 50 (C7), pp.C7-39-C7-66. 10.1051/jphyscol:1989705 . jpa-00229678

HAL Id: jpa-00229678 https://hal.science/jpa-00229678

Submitted on 1 Jan 1989

HAL is a multi-disciplinary open access archive for the deposit and dissemination of scientific research documents, whether they are published or not. The documents may come from teaching and research institutions in France or abroad, or from public or private research centers.
L'archive ouverte pluridisciplinaire HAL, est destinée au dépôt et à la diffusion de documents scientifiques de niveau recherche, publiés ou non, émanant des établissements d'enseignement et de recherche français ou étrangers, des laboratoires publics ou privés. 
PHYSICAL PROPERTIES OF ULTRA THIN FILMS STUDIED BY X-RAY OPTICAL TECHNIQUES LANGMUIR-BLODGETT MULTILAYER STRUCTURES; ORGANIC MONOLAYERS ON WATER AND THE SPREADING OF POLYMER MICRO-DROPLETS

\author{
J.J. BENATTAR, J. DAILLANT, L. BOSIO*, (1) and L. LEGER* * ( 2 ) \\ DPhG/SPSRM, CEN-Saclay, F-91191 Gif-sur-Yvette Cedex, France \\ * Laboratoire de Physique des Liquides et d'Electrochimie, ESPCI, 10 rue \\ Vauquelin, F-75321 paris Cedex 05, France \\ * physique de la Matière Condensée, Collège de France, 1 place \\ Marcellin-Berthelot, F-75231 Paris Cedex 05. France
}

Résumé - Cet article a pour objectif de montrer comment l'optique $X$ et plus particulièrement $1 a$ réflectivité des rayons $X$ aux incidences rasantes peut être appliquée avec succès à l'étude de problèmes physiques très différents faisant intervenir les films ultra minces.

Premièrement, sur un système de couches de Langmuir-Blodgett, nous analysons 1a contribution des interférences produites par les interfaces externes comparée a celle du film.

Deuxièmement, nous présentons une étude des ondes capillaires excitées thermiquement sur une monocouche d'acide béhénique à la surface de 1'eau, par mesure de la rugosité en fonction de la pression de surface. Nous avons observé une forte chute de la rugosité à la transition liquide condensé-solide. Ce comportement remarquable est attribué à l'effet de la rigidité de courbure qui apparaît en phase solide.

Enfin, nous décrivons l'étalement de microgouttes de polymère sur des surfaces solides bien caractérisées. Nous avons trouvé un comportement très général dans lequel la majeure partie du volume du polymère est contenue dans une langue d'épaisseur moléculaire qui s'étale en avant de la partie centrale. La cinétique et les étapes finales de l'étalement ont été déterminées.

Abstract - In this paper we show how X-ray optics and in particular X-ray reflectivity at glancing angles can be applied successfully to study very different kinds of physical problems involving ultra thin films.

First, we have analyzed the relative contributions of the external interfaces and of the internal structure of a Langmuir-Blodgett multilayer system to the rich interference pattern.

(1) Coliaboration in the atudy of monolayers on water.

${ }^{(2)}$ Collaboration in the study of the spreading of polymer droplets. 
Secondly, we have investigated thermally induced capillary waves on a monolayer of behenic acid on water and measured the corresponding roughness as a function of the surface pressure. We observed a strong decrease in the roughness at the liquid condensed to solid phase transition. This significant and striking feature is attributed to the appearence of a bending rigidity in the solid phase.

Finally, we have studied the spreading of polymer micro-droplets on well characterized solid surfaces. We have found a very general behavior in which the majority of the polymer volume is contained in a tongue whose thickness is on the order of the molecular lateral size that spreads ahead of the central part. The kinetics and the final stages of the spreading are determined.

\section{1 - INTRODUCTION}

In the last several years, different techniques using $X$-rays at glancing angles such as reflectivity[1], surface diffraction[2] and fluorescence[3] have been shown to play an increasingly important role in the investigation of interfacial phenomena and, more particularly, in the field of physical chemistry. The progress in these new $X-r a y$ techniques is due both to an increasing precision of diffractometer displacements (currently, $10^{-3}$ or even $10^{-4}$ degrees is easily achievable) and to a development in the basic concepts and in the treatment of the experimental data (application of the optical formalism and consideration of spatial coherence of X-rays): These new X-ray optical techniques compliment such classical optical techniques as ellipsometry and light scattering.

In this review we describe a series of investigations concerning different physical systems which show the evolution in our use of this tool.

First the study of Langmuir-Blodgett (L.B.) multilayers highlighted the important role of interfaces in describing the rich interference patterns measured in these systems. Secondly, a measurement of the surface roughness of a Langmuir monolayer on water yielded a value for the bending rigidity which appears at the liquid condensed to solid phase transition. Finally, we present a very oxiginal use of X-ray reflectivity to study the shape of micro-droplets spreading on smooth, solid surfaces in order to elucidate the wetting by polymers on a microscopic length scale.

Using Langmuir-Blodgett multilayer films composed of a small number of organic layers (with a total thickness not exceeding few hundred Angströms), we have shown.that many striking interference patterns can occur around the Bragg peaks ool (corresponding to the periodicity of the layers). These patterns have put in evidence the important role played by the interfaces and especially by the substrate/film and film/air diopters. The reflections from these interfaces give rise to interferences (the so-called Kiessig fringes) which can strongly modify the subsidiary maxima of the Bragg peaks, thus giving an absolute reference for phase determination. This will be further detailed below.

It is of interest to combine X-ray reflectivity, which yields the profile of the electron density perpendicular to the surface, with another $\mathrm{X}$-ray technique which characterizes the in-plane order. In the case of very thin films deposited or grafted on solid substrates, this problem is rather difficult to solve and requires the use of a very specialized technique such as surface diffraction at grazing. incidence[4]. In this technique, a highly collimated beam strikes the surface at incidence just below the critical angle $\theta_{c}$ thus giving rise to an evanescent wave propagating parallel to the surface. One then measures the diffraction of this evanescent wave by planes normal to the surface. Diffuse scattering by the substrate is suppressed by choosing an angle of incidence such that the beam is totally reflected by the substrate. This is very easy to 
realize in the case of thin organic films on denser substrates such as silicon.

Another way to investigate the in-plane order is to use a transmission geometry with a specific substrate yielding negligible, diffuse scattering. These two methods shall be illustrated below.

Depending on the complexity of the physical system studied, one can extract many different details from the determination of the complete electron density profile. In the case of a complex system, such as the above mentioned multilayers, one can obtain only purely structural information (periodicity, average densities and interfacial roughness). In the case of a monolayer of behenic acid on water, the purpose was to measure the roughness due to thermally induced capillary waves as a function of the surface pressure, particularly at the liquid condensed to solid phase transition. We shall show that, for such a simple system, roughness and structural aspects of the electron density are largely uncorrelated. Here, the physical interest lies principally in the determination of the roughness. In the case of the spreading of polymer micro-droplets on solid surfaces, the determination of the change in the shape of the droplet (i.e. the evolution of the thickness and the density at each point of the droplet) is much more important.

Another, more technical, aim of this paper is to demonstrate the feasability of very accurate reflectivity and surface diffraction experiments on thin organic films (from a few Angströms to hundreds of Angströms) without the necessity of the brightness of a synchrotron source but, rather, by using a simple, conventional X-ray tube. Nevertheless, one must note that very specialized surface diffraction experiments such as the study of surface reconstruction[5] of atomic monolayers requires the higher flux of a synchrotron source. For most other cases, the use of an X-ray tube has many advantages compared to the synchrotron. All orientations of the beam may be used; in particular, it is quite easy to displace the X-ray source for studies of liquid surfaces (or any other geometry in which the sample must remain fixed). These considerations led us to design a special 4-circle diffractometer optimized for interface studies that resulted from a synthesis of many technical ideas separately developed in different laboratories (surface diffraction and fluorescence at grazing incidences ${ }^{[6]}$ and reflectivity ${ }^{[7]}$ ).

\section{2 - EXPERIMENTAL APPARATUS}

First we shall describe the two different diffractometers used to carry out the experiments reported in this paper, one $(\theta-\theta)$ for the monolayer on water studies, and another (4-circles) for the solid surface studies.

(a) The diffractometer for liquids

The principal requirement of such a diffractometer is a $\theta-\theta$ geometry which keeps the sample horizontal (here a liquid). The simplest solution has the X-ray tube and, of course, the detector moving axound a common horizontal axis. This experimental device is schematically represented in Figure 1 and detailed in ref. [7]. 


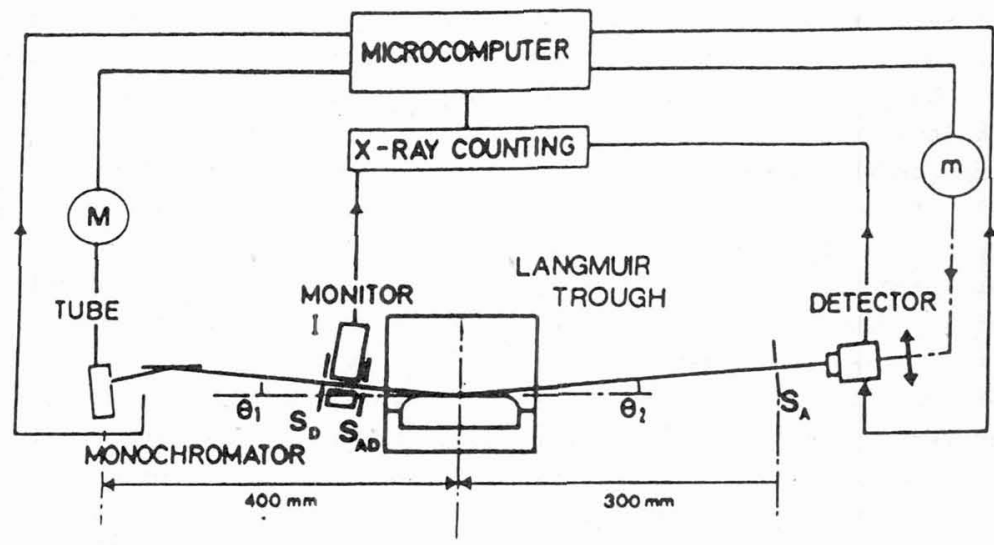

Fig. 1. Diffractometer for liquids.

The Cuko, radiation is selected using a flat LiF(200) monochromator under vacuum

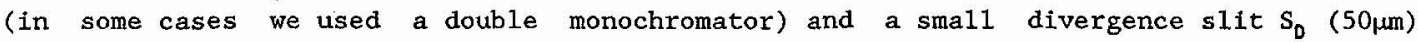
followed by an antidiffusion slit $(0.25 \mathrm{mrad})$. The intensity of the incident beam $\left(I_{0}\right)$ is monitored by an ionization chamber $I_{c}$ and that of the reflected beam $I$ detected by a scintillation counter placed behind the analysis slit $S_{A}(50 \mu \mathrm{m} \times 10 \mathrm{~mm})$. The angular accuracy of the diffractometer is $\pm 0.05 \mathrm{mrad}$. In general, a reflectivity curve is recorded by scanning the reflected beam at each incident angle, to determine its profile and to measure the background which is then subtracted.

(b) The 4-circle diffractometer for solid surfaces

This 4-circle diffractometer was assembled using commercially available elements. It was designed in our laboratory and built in collaboration with Micro-Contrôle, in Evry, France. It is represented in Figure 2. The diffractometer allows

(a)

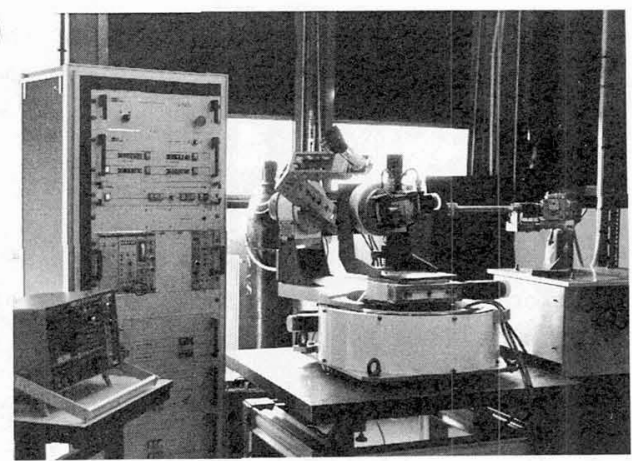

(b)

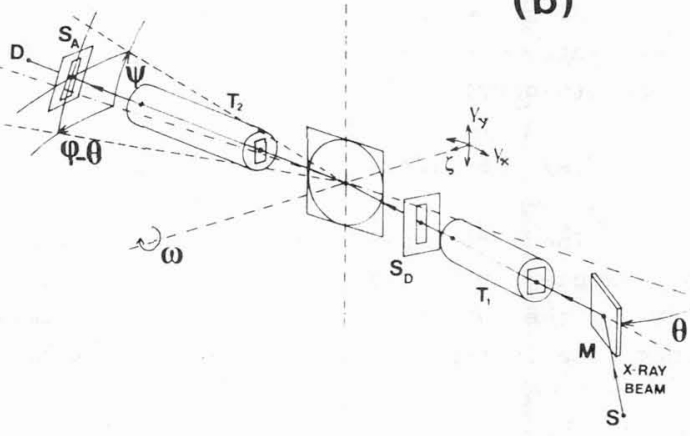

Fig. 2. (a) 4-circle diffractometer for solid surfaces.

(b) Schematic representation of the geometry. 
not only reflectivity measurements, but also grazing incidence surface diffraction and fluorescence studies. The technical ideas which led to the conceiving of this diffractometer came from different origins : for example, from the 4-circle diffractometer used for surface diffraction and fluorescence at grazing incidence described in reference [6] and from the 2-circle diffractometer used for X-ray reflectivity of reference [7]. The $X$-ray optical techniques require a very high accuracy in the incident angles, achievable using stepping motors with an elementary displacement of $10^{-3}$ degree.A conventional, fine-focus copper tube was used as an X-ray source. The monochromater is a LiF(200) which selects the CuK $\alpha_{1}$ line. The low divergence ( $\left.0.1 \mathrm{mrad}\right)$ is obtained by means of a small slit $\left(S_{D}=25 \mu \mathrm{m}\right.$ wide) placed at $40 \mathrm{~cm}$ from source. A slit $S_{H}\left(100 \mu \mathrm{m}<S_{H}<5 \mathrm{~mm}\right)$ can be used to limit the width of illuminated area on the sample. The scattered beam is detected either by a scintillation counter, a linear sensitive detector or a Sili detector. In the

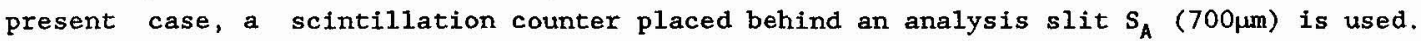
Note that the sample to detector distance is also $40 \mathrm{~cm}$. The incident and reflected beams pass through vacuum flight paths.

A goniometric head $\left(\gamma_{x}, \gamma_{y}\right)$ and a translation $(z)$ are used to position the surface of the sample at the center of the diffractometer parallel to the beam sheet at $\theta=0$.

This 4-circle diffractometer was used for the study of $L . B$. films and the spreading of droplets on silicon wafers. Thus, the silicon wafers are held vertically but, as the droplets are very thin, they are not affected by the gravity.

The main results presented here have been obtained by reflectivity experiments. Surface diffraction experiments are shown essentially to illustrate the potential of this method. They concern a L.B. film and the silane layer grafted on silicon used as a low energy substrate for the spreading study. In these experiments, $\theta$ is fixed to an angle smaller than the critical angle $\theta_{c} \cdot \varphi$ and $\psi$ have to be moved to explore the Bragg rods. In the case of a two-dimensional powder, $\omega$ remains fixed (Figure $2 b$ ).

\section{III - STUDY OF IN-PLANE ORDERING}

For such an investigation, the scattering vector $\vec{q}=\vec{k}_{F}-\vec{k}_{1}$ should have its main component lying parallel to the surface. Two kinds of experiments can provide such a geometry : diffraction in transmission (Figure $3 a$ ) and surface diffraction at glancing angles (FIgure $3 b$ ).

(a)

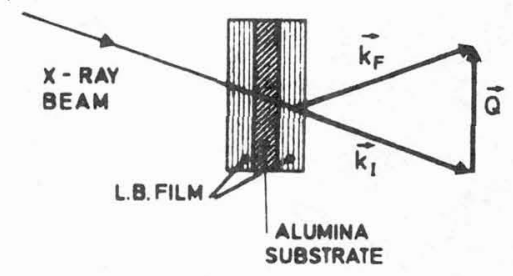


(b)

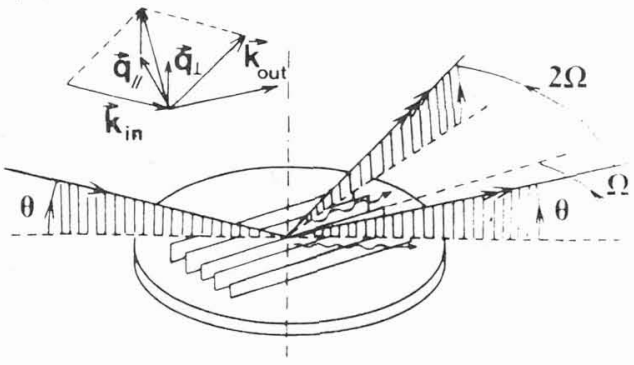

Fig. 3. (a) Transmission geometry, and (b) Surface diffraction.

a) Diffraction in transmission

Only a few complete structural studies have been performed on organic thin films mainly due to their rather small thickness (giving rise to a small X-ray diffraction signal of the film compared to that of the substrate). In a previous paper, we presented a new method[8] which was applied to a Langmuir-Blodgett film of a charge transfer complex (N-docosylpyridinium, tetracyano-quino-dimethane).

Although free-standing films of such a system cannot be used due to the fact that substrate is essential to build up the layers, this difficulty can be circumvented by using a very thin, weakly adsorbing micro-crystallized film as a substrate. A $1000 \dot{A}$ thick film of alumina stretched onto a flat ring yielded negligible $X$-ray diffuse scattering. As the films are very thin $(\sim 500 \AA)$, the scattered intensity is also very weak. Thus, to improve the signal-to-noise ratio, the entire diffraction camera is maintained under vacuum. Figure $4 \mathrm{~b}$ shows a X-ray pattern which exhibits numerous and homogeneous sharp (hko) xings. Some simple conclusions can be drawn from these results. First, the existence of rings gives direct evidence of a polycrystallinity within the layers. Moreover, when the sample is rotated, few hke rings appear (Fig.4a); this clearly means that the layers are rather correlated forming a three dimensional ordered structure.
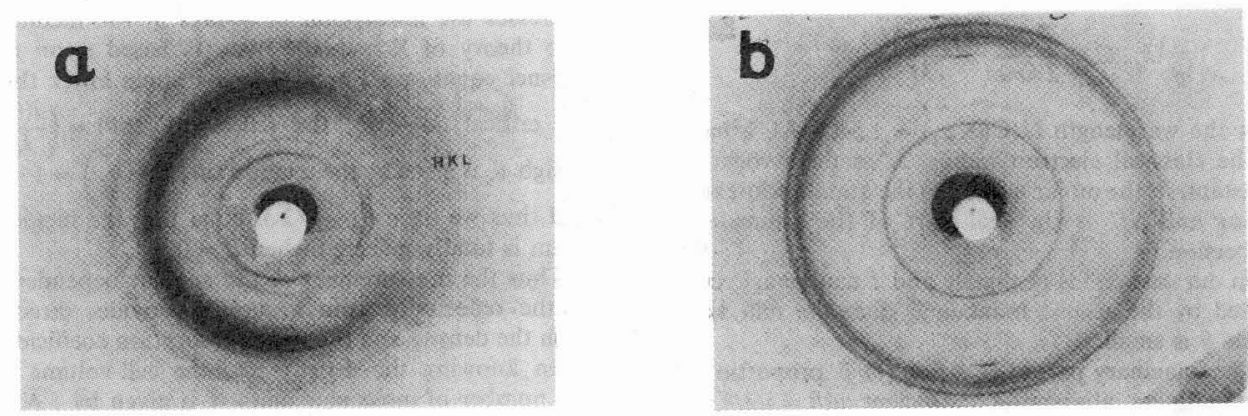

Fig. 4. X-ray transmission patterns; (a) oblique : hk $\ell$ reflections, and (b) Perpendicular : hko. 
The indexation of the set of hko reflections gives the parameters of the cell basis. We found $a=12.8 \AA, b=77 \AA$ and $r=123.5^{\circ}$; the $c$ parameter has been easily obtained from the oo $\ell$ reflections. Using these parameters combined with chemical and steric considerations, we were able to propose a complete realistic structural model for this system.

\section{(b) Diffraction at grazing incidence}

These experiments are not directly related to the basic purpose of this paper, but it is interesting to show an example of what can obtained with a single organic layer. The compound here is behenic acid deposited on a silicon wafer. Another experimental example will be given later in the text.

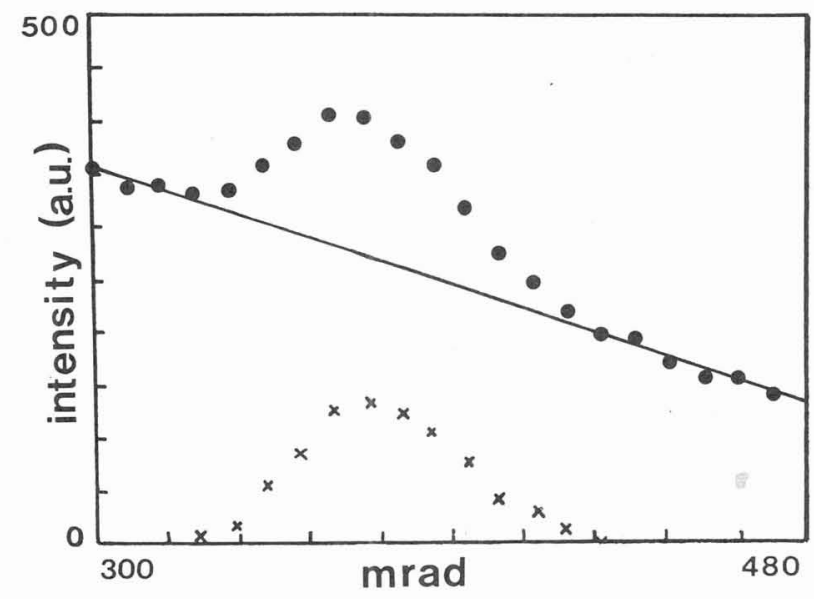

Fig. 5. Surface diffraction from a single organic layer deposited on silicon.

Figure 5 displays the surface reflection at large angles corresponding to the distance within the layer between neighbouring molecules ( $4.8 \AA$ ). It is interesting to notice that this curve was recorded in. several hours using a fine focus copper tube at $1200 \mathrm{~W}(40 \mathrm{kV}, 32 \mathrm{~mA})$. In such experiments, it is easy to get a reasonable good signal to noise ratio since the critical angle of the substrate $\theta_{s}$ is much greater than that of the film. For angles less than $\theta_{s}$, the diffuse scattering due to the substrate approximately corresponds to that of the $20 \AA$ thick layer of silica which covers the silicon.

of course, the surface diffraction experiments present disadvantages. In particular, since the projection of the beam on the sample is very large ( $\sim$ few $\mathrm{mm})$, the resolution is poor compared to that of a classical transmission experiment. 
IV - X-RAY REFLECTIVITY

In this section, we shall describe two different ways to analyze a reflectivity curve : by means of the optical formalism and through the use of the Fourier transform with specific conditions on its validity.

Let us first recall that, in specular reflectivity experiments, the incident beam strikes the surface at an angle $\theta$ and the intensity is recorded in the plane of incidence at an angle $\theta$. Thus, the scattering vector $\vec{q}$ is perpendicular to the surface and the experiment gives information about the mean electron density along the normal $z$ (Figure 6).

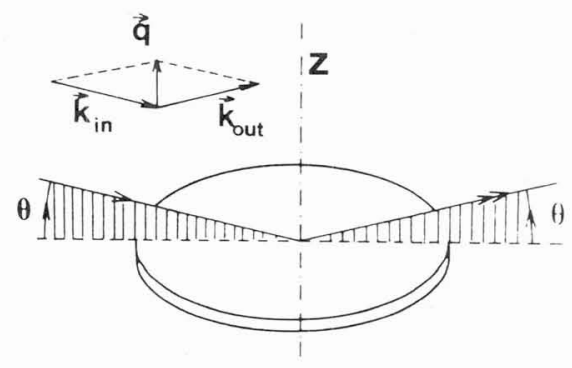

Fig. 6. Reflection geometry.

It should be noted that the coherence of the beam is typically a few $\mu \mathrm{m}^{2}$, i.e. smaller than the illuminated area. Thus, the system behaves like a set of independant subsystems.

The electron density profile is directly related to the refractive index profile along $z$ by the well known relation :

where

$$
n(z)=1-\delta(z)+i \beta(z)
$$

$$
\delta(z)+i \beta(z)=\frac{\lambda^{2}}{2 \pi} r_{e} \sum_{i} f_{i} N_{i}(z)
$$

$f_{i}$ is the complex atomic scattering factor, $\lambda$ the wavelength, $r_{e}$ the classical electron radius and $\mathrm{N}_{i}(z)$ the atomic density. If absorption is neglected as well as the angular dependence of the scattering factor, then $n(z)$ can be written $: n(z)=1-\delta(z)$ with $\delta(z)=\frac{\lambda^{2}}{2 \pi} r_{e} \rho_{e}(z)$, where $\rho_{e}(z)$ is, the electron density along the $z$-axis.

(a) Matrix formalism

Reflectivity calculations that are valid at all angles including grazing inciden- 
Such a method consist of treating the system as a succession of homogeneous laminae. Each lamina is characterized by a transfer matrix $M_{j}$ depending on three parameters : its thickness $d_{j}$, the refractive index $n_{j}$ and the incident angle $\theta, M_{j}$ is given by:

$$
M_{j}=\left(\begin{array}{l}
\cos \psi_{j}-\frac{i}{p_{j}} \sin \psi_{j} \\
-i p_{j} \sin \psi_{j} \cos \psi_{j}
\end{array}\right)
$$

where $\psi_{j}=\frac{2 \pi}{\lambda} n_{j} d_{j} \sin \theta$ and $p_{j}=n_{j} \sin \theta$. The last expression is valid for s-polarization but, in the range of angles we consider, the reflectivity for both the polarizations $s$ and $\mathrm{p}$ are equivalent.

The medium is then represented by a transfer matrix which is the product of the matrices of the constituting laminae. Note that for layered systems such as L.B. films, a natural intermediate stage of this calculation is the evaluation of the matrix of one layer. The reflection coefficient is given in terms of the matrix element $m_{i j}$ :

$$
r=\frac{\left(m_{11}+m_{12} p_{s}\right) p_{0}-\left(m_{21}+m_{22} p_{s}\right)}{\left(m_{11}+m_{12} p_{s}\right) p_{0}+\left(m_{21}+m_{22} p_{s}\right)}
$$

where $p_{s}=n_{s} \sin \theta, p_{0}=n_{0} \sin \theta$ and $n_{0}$ and $n_{s}$ are the indices of air and the substrate respectively.

The final reflectivity is then :

$$
R(\theta) \equiv \frac{I(\theta)}{I_{0}}=|r|^{2}
$$

This method or equivalent computation schemes have been used previously in many reflectivity studies using $\mathrm{X}$-rays or neutrons.

(b) Fourier transforms

In many practical cases, for example organic monolayers deposited on solid surfaces, the first part of a reflectivity curve corresponding to total external reflexion (at very glancing angles, typically about 2 or 3 mrad) does not contain much useful physical information. Thus, it is only necessary to take into account the part of experimental reflectivity curve that corresponds to incident angles sufficiently greater than $\theta_{c}$. In that case one enters the range of application of kinematical theory; the equivalent of the first Born approximation in quantum-mechanical theory becomes valid since multiple scattering may be neglected.

The relation between the reflectivity and the electron density profile is thus related to a simple Fourier transform $R(q)=\frac{1}{\rho_{\infty}}\left|\frac{4 \pi r_{e}}{q} i \int_{-\infty}^{0} \rho(z) \exp (i q z) d z\right|^{2}$ where $q=\frac{4 \pi}{\lambda} \sin \theta=(\vec{q})_{2}$.

After integration by parts one obtains :

$$
q^{4} R(q)=\left(4 \pi x_{e}\right)^{2} \cdot \frac{1}{\rho_{\infty}}\left|\int_{-\infty}^{0} \frac{d \rho(z)}{d z} \exp (i q z) d z\right|^{2}
$$

This expression makes it clear that a reflectivity curve results from the interferences due to large density gradients. 
It should be pointed out that although the matrix formalism is much more general and also very well suited for computations, it obscures the physical origins of the interference effects. The Fourier transform method is less general, but enables analytic calculation and easy discrimination between the origins of the pattern features.

Let us apply such an approach to a very simple and general case of an organic multilayer film in order to show how it is possible to extract different and uncorrelated information from an experimental reflectivity curve.

(c) X-ray reflectivity of Langmuir-Blodgett structures

The reflectivity patterns of all the multilayer films are quite similar, and the experimental curve can be divided in three parts : the total reflection, the small angles and the higher angles. The main differences between the different multilayers concern the interference phenomena occuring around the very first Bragg peaks (ool).
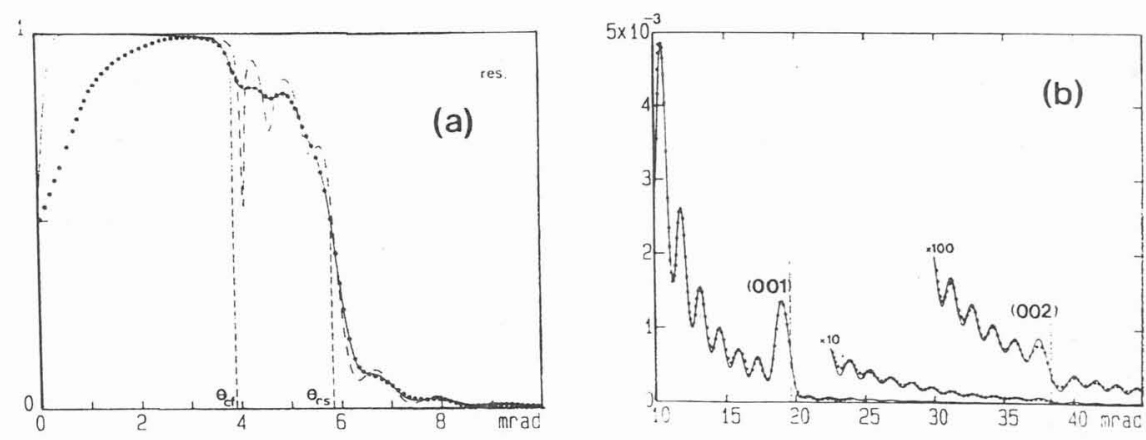

Fig. 7. 27 layer film reflectivity curve (a) Grazing incidences (b) ool Bragg peaks and Kiessig fringes.

- Grazing angles

- The first part of this reflectivity curve (Fig. 7a) ranges between 0 and 10 mrad. For these angles, the film may be considered homogeneous with a mean refractive index $n_{F}=1-\delta_{f}-\beta_{f}$. Measurements of the two critical angles for the film and the substrate $\left(\theta_{c_{f}}=\sqrt{2 \delta_{f}}\right.$, and $\theta_{c_{s}}=\sqrt{2 \delta_{s}}$, respectively) and the shape of the reflectivity curve at low angles (which depends mainly on absorption) allow the determination of film and substrate mean complex indices. Fringes of equal inclination (Kiessig fringes) are already visible, but their shape is greatly altered by the instrumental resolution.

- Interference structure around the first Bragg peaks

- The second part of the reflectivity curve (Figure $7 \mathrm{~b}$ ) is the most interesting since it exhibits a rich interference pattern. Let us first consider the (001) Bragg peak surrounded by subsidiary maxima. 
One can notice that the intensities of the subsidiary maxima are asymmetric axound the first peak. This cannot be explained by regular damping with increasing incidence, since there is about one order of magnitude between the intensities. Actually, these particular effects result from an interference phenomena between waves coming from two origins. First, the waves reflected at the two interfaces air/film and film/substrate would give rise, if alone, to Kiessig fringes. Secondly, waves coming from the finite-size stratified structure which yield Bragg peaks with secondary maxima. Hence, subsidiary maxima and minima are a resulting pattern between Kiessig fringes and secondary Bragg peak maxima. The phase difference between the two beams depends on the relative position of interfaces and stratification (i.e. the film boundaries).

- Optical Mode1

- Using the formalism, the reflectivity curve can be calculated if the index profile $\delta(z)$ is known. The reflectivity curve, calculated using the $\delta(z)$ profile of Figure 7c (the solid line in Figure $7 \mathrm{a}, \mathrm{b})$, is found to fit the experimental data after convolution with the instrumental resolution. The fitted values found for the parameters contained in $\delta(z)$ are close to the theoretical ones, and a re calculated by taking into account the steric hindrance, the atomic composition and the standard distances between atoms.
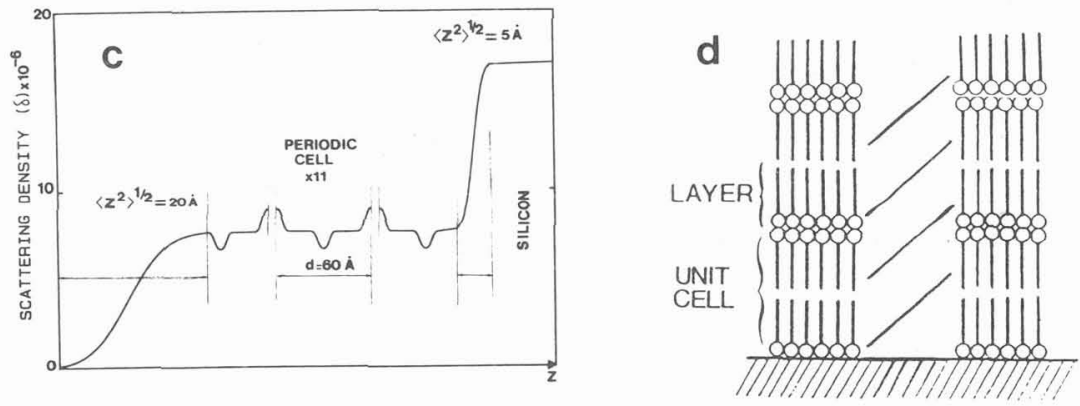

Fig. 7c. Density profile of the 27-layer film.

Fig. 7d. Odd number multilayer film.

- Calculation of the interference structure (case of an odd number of layers)

- In this section we will show an example of an analytic calculation which gives an account of the striking features of the interference around the Bragg peaks. A detailed study is given in ref.[10]. As the system consists of a stacking of $N$ bilayers and a single additional layer, this case corresponds to a non purely periodic structure. The additional (and last deposited) monolayer can be replaced by an homogeneous lamina having the mean index of the film. This affects the phase between reflected waves but can be neglected in the intensity. Under this assumption, the reflectivity can be easily calculated and one finds : 


$$
\begin{aligned}
R(q) & =\left(\frac{8 \pi^{2}}{\lambda^{2} q}\right) \times \frac{\left(\delta_{s}-2 \delta_{f}\right)^{2}}{q^{2}}+\frac{4 \delta_{f}\left(\delta_{s}-\delta_{f}\right)}{q^{2}} \cos ^{2}\left(\left(N+\frac{1}{2}\right) \frac{q d}{2}\right)+F^{2} \frac{\sin ^{2}\left(N \frac{q d}{2}\right)}{\sin ^{2}\left(\frac{q d}{2}\right)} \\
& +2 F \frac{\sin \left(N q \frac{d}{2}\right)}{\sin \left(q \frac{d}{2}\right)} \times \frac{1}{q}\left(\left(\delta_{f}-\delta_{s}\right) \sin \left(\frac{(N+1) q d}{2}\right)+\delta_{f} \sin \left(\frac{N q d}{2}\right)\right)
\end{aligned}
$$

where the index profile is represented by a periodic function between $z=0$ and $z=-N d$ of bilayer spacing d. $\delta_{f}$ is the mean value of the index within the film, $\delta_{s}$ that of the substrate; $F(q)$ is defined as :

$$
F=\int_{-d / 2}^{d / 2}\left[\delta(z)-\delta_{f}\right] \exp (i q z) d z
$$

The value of $q d / 2$ corresponding to a (ool) Bragg peak is $\ell \pi$. It can be seen that the last interference term is the sum of two opposite terms for even $\ell$ and the difference of these terms for odd $\ell$. The absolute values of these terms are small in one case (even l) but large in the other (odd $\ell$ ).

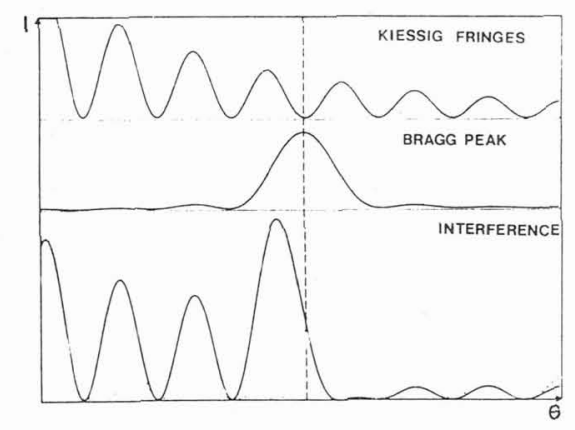

Fig. 8. Decomposition of the interference pattern around 001 Bragg peak.

Figure 8 shows the interference effect around the (ool) Bragg peak. In this case, fringes and secondary maxima coincide, so that the interference has a very large effect on the intensity of the subsidiaries. The sign change of the large interference term from one side of the Bragg peak to the other results in a very strong damping of the fringes. The ratio of their intensities before and after the peak is actually about 100 .

It is interesting to note that the last interference term in the expression of the reflectivity can provide information about the phase of the scattered radiation. This problem has been treated in ref.[11] where we have demonstrated that the waves reflected from both interfaces of a thin film (or only one of them) may provide a reliable reference for phase identification. 


\section{$V$ - X-RAY REFLECTIVITY STUDY OF A MONOLAYER AS FUNCTION OF SURFACE PRESSURE}

Due to the very simple chemical structure of fatty acids, L.B. films built up with these molecules are model systems for the study of very complex interference patterns, i.e. to extract detailed structural information. On the other hand, the structure of a monolayer is transparent, and thus attention can be focused on a refined picture of the interfaces. This illustrates the wide potentials of X-ray reflectivity.

of course new problems need to be solved to study these monolayer directly on water. First of all, the very small amount of diffracting matter, i.e. a molecular monolayer, implies that the experiments are long, but it should be pointed out that this study does not necessarily require the brightness of a synchrotron source since the limitation of this experiment is only due to the small signal-to-"noise" ratio at high angles (the signal scattered by the monolayer becomes very small compared to the intensity diffusely scattered by the bulk).

Monolayer of amphiphilic molecules have been widely studied with an aim to characterize the phase transitions. These systems provide simplified models of biological membranes and have been the subject of many investigations by means of different optical techniques $[12,13]$. Diffraction at grazing incidence[14,15] as well as X-ray reflectivity have also been employed[16]. The dynamical aspects of capillary waves have been intensively studied by ellipsometry[18] and $X$-ray reflectivity[20,21] on simple 1iquids and on interfaces with a surfactant between two liquids and that of viscoelastic properties by diffuse light scattering[19].

Using $\mathrm{X}$-ray reflectivity we were able for the first time, to demonstrate the appearance of a non-zero bending elasticity at the liquid-condensed to solid phase transition of a simple fatty acid and we have measured its magnitude[17]. Moreover, the proved that exclusive use of the capillary wave model alone to describe the surface roughness. Besides the refined structural information studied on the same chemical systems (L.B. films), these experiments account for the versatility of the techniques.

\section{(1) Experimental}

For this study of monolayers on water, we chose behenic acid $\left(\mathrm{CH}_{21} \mathrm{H}_{43} \mathrm{COOH}\right)$, rather than a more complex ionized species. The $\mathrm{pH}=5.5$ (due to a $\mathrm{CO}_{2}$ adsorption) remained constant throughout the experiment, performed at room temperature $\left(22 \pm 1^{\circ} \mathrm{C}\right)$.

Details of our Langmuir trough $[17]$ are schematically represented in figure $9 a$. The surface pressure $\Pi$ ( $\Pi=\gamma_{W}-\gamma, \dot{\gamma}_{w}=72 \mathrm{mN} / \mathrm{m}$ for pure water) is provided by a movable barrier whose displacement is controlled by means of a Wilhelmy balance. The level of the water surface is adjusted by means of a differential photodiode to within $\pm 5 \mu m$ through the heating of an auxiliary tank providing extra water. The entire device is maintained under a nitrogen flow.

The surface pressure-area isotherm (Figure 9b), recorded using a trough device (Figure 9a), displays the usual break in the slope at $\Pi \simeq 20 \mathrm{mN} / \mathrm{m}$ corresponding to a liquid condensed to solid transition (LS transition) [22].

All the experiments were performed with the diffractometer for surface studies described previously. 


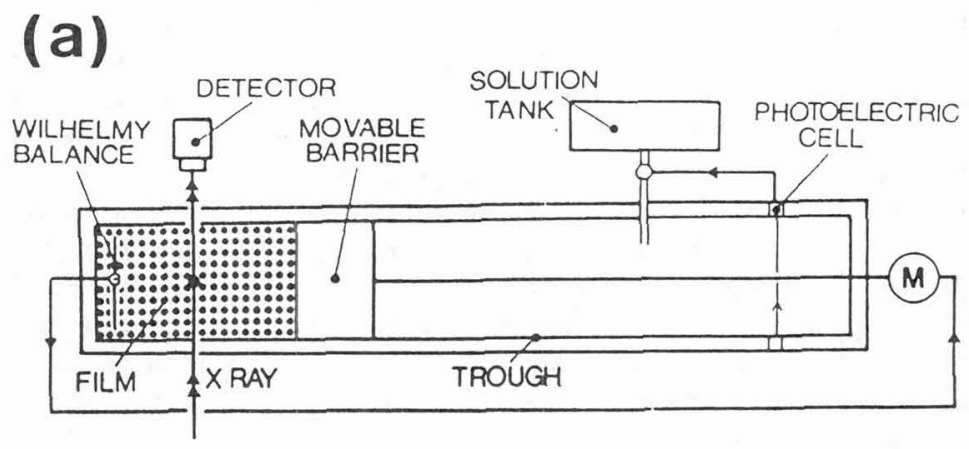

\section{(b)}

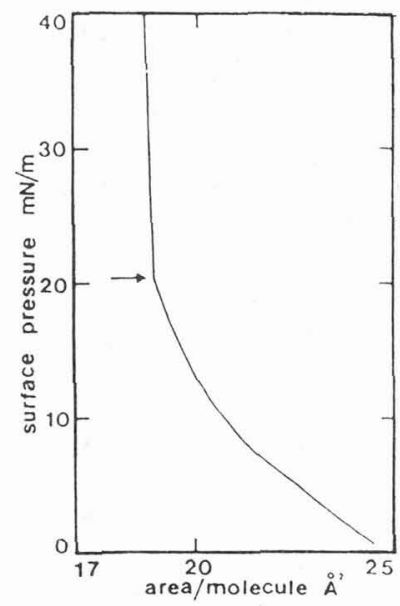

Fig. 9. (a) Schematic representation of the Langmuir trough. (b) Surface pressure-area isotherm

(2) Results

\section{(a) Reflectivity curves}

Two representative experimental reflectivity curves are reported in Figure 10 . Figure 10a was recorded in the liquid condensed phase and Figure $10 \mathrm{~b}$ in the solid phase. Both exhibit a well-defined first-order peak (or fringe) corresponding to the interference between beams reflected at the two interfaces : film/air and film/water. Note that the peak at $\Pi=22 \mathrm{mN} / \mathrm{m}^{2}$ is twice as high as at $\Pi=15 \mathrm{mN} / \mathrm{m}^{2}$ and that the curve of Figure $10 \mathrm{~b}$ is shifted to the left due to an increase in the monolayer thickness related to a decrease in the molecular tilt angle. 

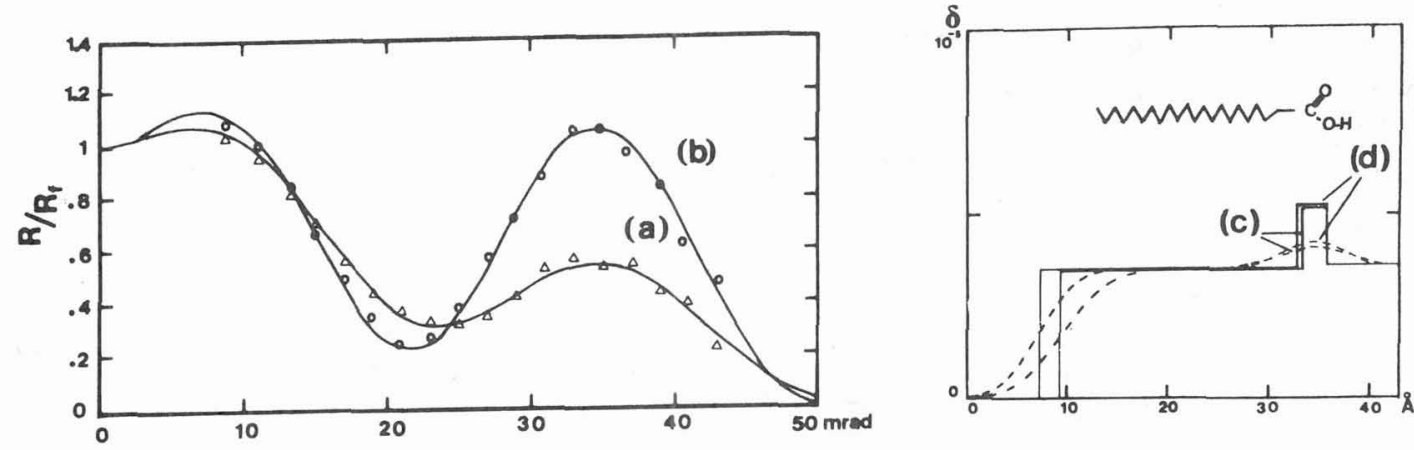

Fig. 10. Reflectivity curves normalized to the Fresne1 reflectivity of water and density models ; (a) and (c) : liquid condensed and (b) and (d) : solid.

Two kinds of separate information can be easily and directly extracted from the experimental curves by means of a simple fit to the $R / R_{f}$ curve : the thickness of the monolayer, using the minimum in the interference pattern (and thus the tilt angle), and the roughness. The calculated curves are convoluted by the resolution of the diffractometer and fitted to the experimental data.

\section{(b) Tilt angle determination}

The behenic acid monolayer can be broken into two laminae : one for the chains and the other for the polar groups. From a structural point of view, we find that when the surface pressure is varied from $5 \mathrm{mN} / \mathrm{m}$ to $45 \mathrm{mN} / \mathrm{m}$, the real part of the index $\delta_{c}$ for the chains does not change significantly $: \delta_{c} \simeq 3.45 \pm 0.1 \cdot 10^{-6}$, whereas the index of the polar group $\delta_{p}$, whose mean value is $5.10 \pm 0.15 \cdot 10^{-6}$, increases slightly with pressure. The main structural changes appear at the LS transition at $\Pi \simeq 20 \mathrm{mN} / \mathrm{m}$. A drop occurs in the tilt value going from $\sim 28^{\circ}$ to $\sim 18^{\circ}$, based upon a molecular length of $27 \AA$ as given by an all trans configuration model (Figure 11a). However, this tilt angle determination is not sufficiently accurate to discriminate between a small tilt angle and strictly zero as well as slight conformational changes. Let us remark that, in Figure 1la, the drop observed at the LS transition does not occur at a well-defined pressure, but spreads over $4 \mathrm{mN} / \mathrm{m}$. This may indicate a first-order transition characterized by a two-phase coexistence region[23]. The observed tilt angle should then result from an average over the tilt angles of the solid domains and of the liquid areas. 


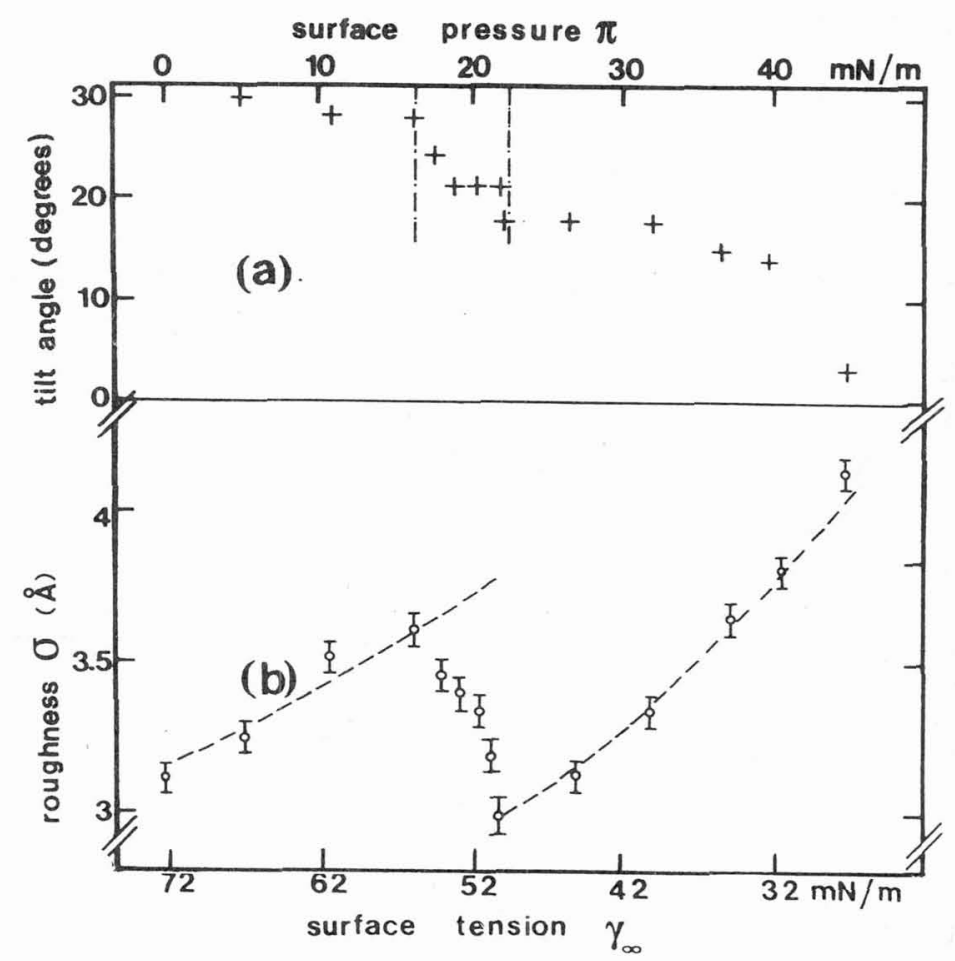

Fig. 11. (a) Tilt angle variation, (b) Measured roughness

\section{(c) Capillary roughness}

Generally, the capillary roughness is described by Gaussian statistics and, in this approximation which is rigorous in the case of weak coupling between modes, the reflectivity can be written as $: R\left(Q_{z}\right)=R_{F}\left(Q_{z}\right) \exp \left[-Q_{z}^{2} \sigma^{2}\right]$ for angles $\gg \theta_{c}(\sim 2.5 \mathrm{mrad})$, where $R_{f}\left(Q_{z}\right)$ is the Fresnel reflectivity of a two-laminae model with perfect plane interfaces (Figure 10c) and $\sigma^{2}$ is the mean-square roughness. In practice, the roughness is directly introduced in the density profile as an error function providing the Gaussian attenuation $[20]$.

The entire set of experimental data is reported in Figure 11. In particular, the variation of the roughness as a function of surface pressure is represented in Figure $11 b$. Each point represents a different experiment on a fresh film after cleaning the trough and changing the water. The different surface pressures were selected at random and remeasurements of the same surface pressures yielded identical values. The most striking feature is the drop in the roughness at the LS transition $(\Pi \simeq 20 \mathrm{mN} / \mathrm{m})$. As also observed for the tilt angle, this transition does not occur at a given pressure, but is spread over $4 \mathrm{mN} / \mathrm{m}$. The observed roughness results from an average of the roughness of liquid areas and of solid domains. During the transition the ratio of solid domains to liquid areas increases and the roughness decreases until it reaches a value corresponding to that of the solid. 


\section{(3) Discussion}

The striking feature which occurs at the liquid condensed to solid phase transition is interesting because it provides a new "signature" of that transition. To understand this behavior, we have used a theory of capillary waves (Ref. [24]) which takes into account the coupling between the modes of fluctuations. In this frame the surface tension $\Psi$ and the bending rigidity $K$ should be functions of the length scale $1 / q$ : $r(q)=\gamma_{\infty}+(3 / 8 \pi) k_{B} T_{q}^{2} \quad\left(\gamma_{\infty}\right.$ is the macroscopic surface tension). The $q^{2}$-term in the expression of $\gamma(q)$ cannot be neglected since the resolution area of the spectrometer includes wave vectors $\mathrm{q}>\sqrt{8 \pi \gamma_{\infty} / 3 \mathrm{k}_{\mathrm{B}} \mathrm{T}} \sim 10^{8} \mathrm{~cm}^{-1}$.

The observable roughness $\sigma_{c}$, whose effect is to reduce the specular intensity, is obtained by integrating the squared amplitude over all the modes which scatter outside of the analysis slit :

$$
\sigma_{c}^{2}=\frac{k_{B} T}{4 \pi^{2}} \int_{q_{x}^{m i n}}^{\infty} \int_{q_{y}^{m i n}}^{\infty} \frac{d q_{x} d q_{y}}{\Delta \rho g+r(q) q^{2}+k(q) q^{4}},
$$

$(0 \mathrm{x}, 0 \mathrm{y})$ is the plane of the liquid surface, $q_{\mathrm{m} i n}$ is directly related to the dimensions of the analysis slit and to the distance to the sample. Note that the gravitational term may be neglected $q_{x}^{m i n}=(\pi / \lambda) \sin \theta\left(\delta_{z} / \ell\right) \sim 1.2 \cdot 10^{3} \mathrm{~cm}^{-1}$ (for $\theta=35 \mathrm{mrad}$ corresponding to the maximum of the reflected intensity and $\mathrm{q}_{y}^{\mathrm{min}}=(\pi / \lambda)\left(\delta_{y} / \ell\right) \sim 6.8 \cdot 10^{6} \mathrm{~cm}^{-1}$, where $\delta_{z}$ and $\delta_{y}$ are, respectively, the width $(50 \mu \mathrm{m})$ and length $(10 \mathrm{~mm})$ of the analysis slit and $\ell$ is the sample-to-detector distance $(300 \mathrm{~mm})$.

The first experimental point in Figure $11 \mathrm{~b}(\Pi=0)$ corresponds to the roughness of bare water: we found $\sigma \simeq 3.1 \AA$. From this point until the transition, one observes an increase of the roughness in good agreement with the theory with $\mathrm{K}=0$. Thus, the sole contribution of capillary waves can account for the measured roughness, unlike as reported previously[20,21]. It does not appear necessary, in the frame of a coupled-mode theory, to introduce a supplementary contribution of an intrinsic profile $\sigma_{p}$ at an arbitrary cut-off. This is due to the fact that the capillary wave model implicitly includes the intrinsic profile $\sigma_{p}$ described as a sum of high wave vector modes if, as here, the integration domain is extended up to infinity.

After the transition, the roughness becomes significantly lower and this effect is due only to the appearance of a rigidity of the solid monolayer; $K$ becomes nonzero and is the only relevant parameter. The first value of the roughness after the Ls transition, in the solid phase, was accurately measured and we found $\sigma=(3 \pm 0.05) \AA$. Using formula ( 1 ) with only one adjustable parameter, the macroscopic bending elasticity constant $K_{\infty}$, we found $\mathrm{K}_{\infty}=190 \mathrm{k}_{\mathrm{B}} \mathrm{T}$ under our resolution conditions. Moreover, one can notice that the experimental uncertainty is compatible with a rather wide of values for the rigidity (for $\sigma=3.05 \AA, \quad \mathrm{k}_{\infty} \simeq 120 \mathrm{k}_{\mathrm{B}} \mathrm{T}$ and for $\sigma=2.95 \AA \mathrm{k}_{\infty} \simeq 300 \mathrm{k}_{\mathrm{B}} \mathrm{T}$ ). This is because of the logarithmic dependence of $\mathrm{K}$ in formula (1). Another interesting conclusion is that $\mathrm{K}_{\infty}$ in the solid phase appears to be nearly independent of pressure.

Finally, one can draw several conclusions from this X-ray reflectivity study of a monolayer; the most interesting result is that the liquid condensed to solid transition is accompanied by the appearance of a bending rigidity. X-ray reflectivity provides an elegant and reliable manner to determine its value. The logical next step, which we have undertaken, is the study of the liquid expanded to the liquid condensed phase transition using an other well known fatty acid (pentadecanoic acid). 


\section{VI - X-RAY REFLECTIVITY STUDY OF THE SPREADING OF POLYMER MICRODROPLETS}

$A$ third and original application of $X$-ray reflectivity that we would like to discuss in this review is the determination of complex electron density profiles in the field of physical chemistry; when studying droplets, a new difficulty arises in comparison with classical $\mathrm{X}$-ray reflectivity studies since the thickness of the film varies over the surface. We have shown that, using a sufficiently collimated beam, it is possible to determine the profile of a droplet[27]. Moreover the detailed information obtained from $X$-ray reflectivity data allows the presentation of a refined picture of ultra thin wetting films. First of all, the independent determination of thicknesses and densities is essential since the exact amount of matter at the surface can thus be determined. Diffusion coefficients can be calculated and mechanisms at a molecular level may be suggested.

We shall illustrate the importance of this new tool for wetting studies by discus sing recent experiments performed in our group which shall be published elsewhere [34].

A large number of practical processes involve the spreading of a liquid on a solid surface; thus considerable work has been carried out on the wetting phenomena. Until recently, few studies have taken into account the role played by ultra-thin films. de Gennes and Joanny [25] considered the role of long range cohesive forces of the liquid and have analyzed their effect on the profile of droplets during the spreading as well as in the case of capillary rise. Moreover, they pointed out that, in the case of non-volatile liquids, the spreading should stop due to a balance between these and the spreading forces. The relevant parameters in the wetting phenomena are the surface tension of the liquid $r$ and the spreading parameter $S$. $S$ measures the energy gained per unit surface during the spreading, and, for $S \geqslant 0$, spreading spontaneously occurs. The equilibrium thickness e depends on $s: e=a \sqrt{\frac{3 \gamma}{2 S}}$ where the dimension of $a$ is on the order of magnitude of the molecular length. We test the validity of this picture. In this study we used two kinds of substrates with $S>0$, one corresponding to a low energy and another to a high energy surface and a liquid polymer whose surface tension is $\gamma \sim 22 \mathrm{mN} / \mathrm{m}[26]$. Thus, as $\mathrm{S} / \gamma$ is never 1 , the classical concepts of a hydrodynamic theory cannot be applied; films of molecular thickness develop and we will show that surface diffusion plays a large role. We present measurements of the shape of the precursor film which spreads ahead of the droplets as well as that of the center of the droplet. As the spreading is very slow, we show the different characteristic times involved by the kinetics and, finally, we describe the final stages of spreading.

\section{(1) Experimental system}

\section{(a) Chemical details}

The polymer polydimethylsiloxane (PDMS) is a non volatile liquid at room temperature. The choice of a polymer is convenient since the viscosity can be varied over a rather wide range by varying the molecular weight, negligably affecting the other characteristics of the liquid. The surface tension of this polymer is $\gamma \sim 21-22 \mathrm{mN} / \mathrm{m}$.

The first type of substrate used in these experiments was an optically polished (400) silicon wafer ( $50 \mathrm{~mm}$ in diameter and $2 \mathrm{~mm}$ thick to ensure a good flatness) covered by its natural oxide layer ( $20 \AA$ thick). A high energy surface is obtained by strong oxidation of the organic impurities through an oxygen flow under $U . V$ radiation [28]. Water spreads on this surface, thus it has a critical surface tension $\gamma_{c}$ larger than $73 \mathrm{mN} / \mathrm{m}$. The value of $\mathrm{S}$, the spreading parameter, is large for this surface (an estimation of $\mathrm{S}$ is 
$\left.S \sim \gamma_{c}-\gamma \geqslant 50 \mathrm{mN} / \mathrm{m}\right)$.

A reflectivity curve and the corresponding electron density profile are given in fig. 12 for this substrate. The measured surface roughness is $\sqrt{\left(z^{2}\right)}=4.2 \AA$, which can vary slightly with each sample.

A second controlled type of substrate is obtained by chemical grafting of a compact monolayer of octadecyltrichlorosilane (OTS), following Sagiv's procedure [29]. The critical surface tension $\gamma_{c}$ of this substrate is of the order of $22-23 \mathrm{mN} / \mathrm{m}$, and, thus, $s$ is smaller or close to 1 , but still positive.

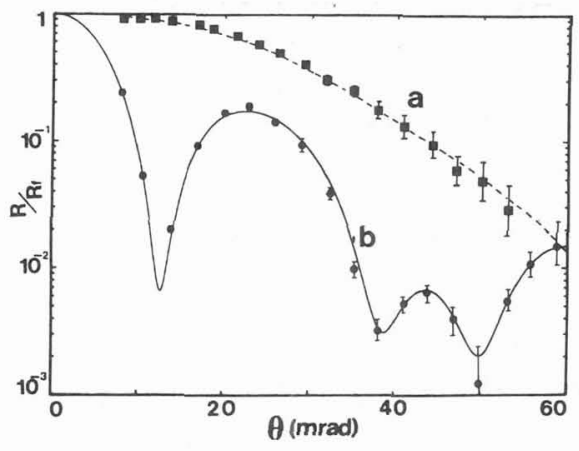

Fig. 12. Fresnel normalized reflectivity curves -

(a) Bare silicon, (b) Silanated wafer.

The corresponding reflectivity curve has been recorded down to an ordonate of $10^{-9}\left(\mathrm{q} \sim 0,65 \AA^{-1}\right)$, providing direct information at a scale on the order of $4 \AA$ (fig. $12 a, b)$. The electron density which yields a good fit to the data clearly contains a denser layer just under the hydrocarbon chains. This layer is attributed to the $S i$ heads of the silane molecules. The roughness of the (air/chains) and (chains/dense layer) interfaces are almost identical (here $\sim 3 \AA$ ), as one might expect for a single molecule. Tidswell et al. have recently studied these surfaces extensively using synchrotron radiation [30] and have given a very precise description of their structure. Note that our data, recorded with a classical $\mathrm{X}$-ray tube, allows a determination just as precise.

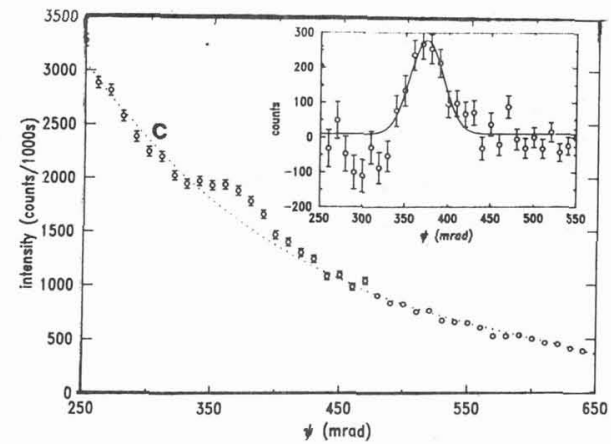

Fig. 12.(c) Surface diffraction from the a $20 \AA$ thick silane monolayer. 
From the surface diffraction data (recorded at an angle of incidence of $\theta=3 \mathrm{mrad})$, we can extract information about the in-plane structure of the grafted layer. Here the position of the Bragg reflection at $q \sim 1.5 \AA^{-1}$ is indicative of a spacing of $d=4.18 \pm 0.04 \AA$ which corresponds to a densely packed hydrocarbon layer (18.5 $\AA^{2}$ /molecule).

\section{(b) Experimental procedure}

Two different geometries have been considered in studies of wetting: the first is simply the spreading of a droplet on a horizontal surface and the second is the capillary rise. These are shown in fig. 13.

In the first type of experiment (fig. 13 a), a droplet is deposited on a baxe or silanated silicon wafer and placed in a small Kapton box to prevent contamination by dust. Since the equilibrium thickness of the film is expected to be on the order of a few Angströms and the surface of the substrate is limited $\left(\sim 20 \mathrm{~cm}^{2}\right)$, we used very tiny droplets $\left(\mathrm{V} \sim 10^{-7} \mathrm{~cm}^{3}\right)$ in order to avoid that they reach the edge of the substrate. The volume was measured by microscopy before beginning the X-ray experiments. The droplets could be studied from right after their deposition until the final stage of wetting, sometimes reached a few months later.

A second type of experiment was undertaken in order to separate the question of the growth of the molecular film from that of the vanishing of the central drop. The polymer was contained in a small reservoir made of Teflon, in which the substrate (silicon wafer) was pinned. The entire system was enclosed in a Kapton box (fig. 13 b).
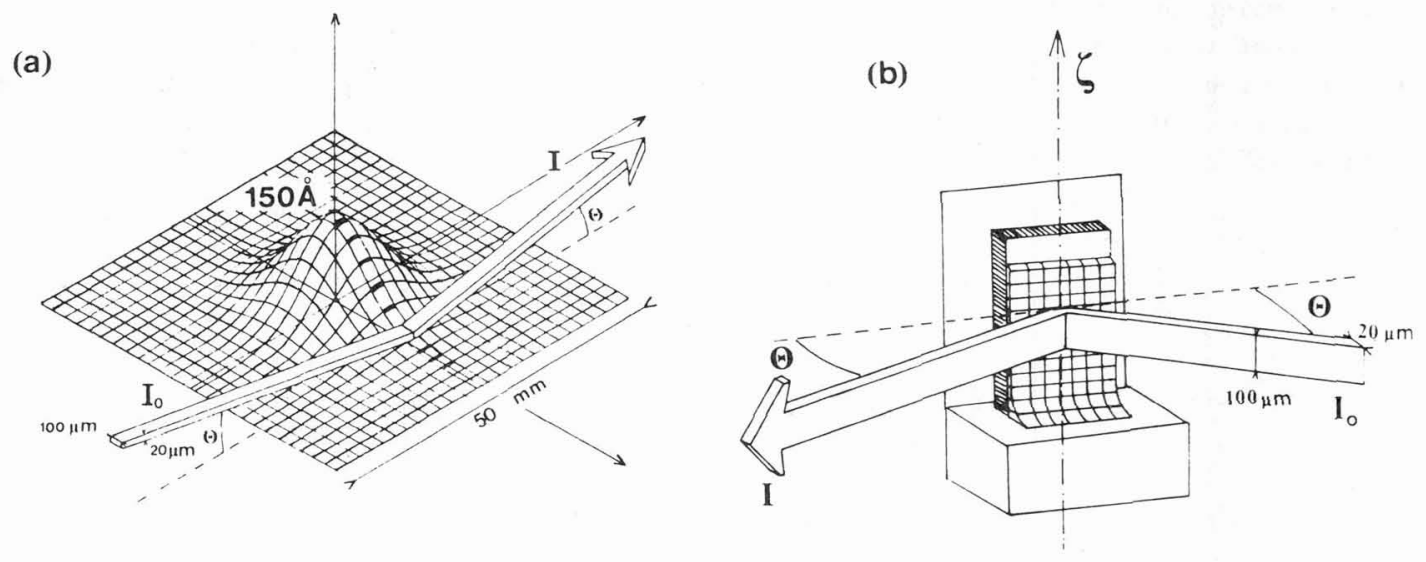

Fig. 13 : Schematic views of X-ray experiments on (a) a droplet and (b) capillary rise 


\section{(2) Results}

The very long time required to reach equilibrium is, in fact, the most serious limitation in these experiments but cannot be avoided with liquids that are clearly non-volatile. Consequently, attention was focused on the spreading of PDMS $6500 \mathrm{~g} / \mathrm{mole}$ on bare silicon. The spreading of this same polymer on silanated substrates appears to be, to a large extent, quite similar. It is worth noting that these systems are expected to cover a wide range of practical situations. Capillary rise experiments have been undertaken to study the growth of the monomolecular film. Higher molecular weight droplets were also studied and the results have been reported elsewhere[34].

(a) Spreading of a droplet of PDMS on bare silicon
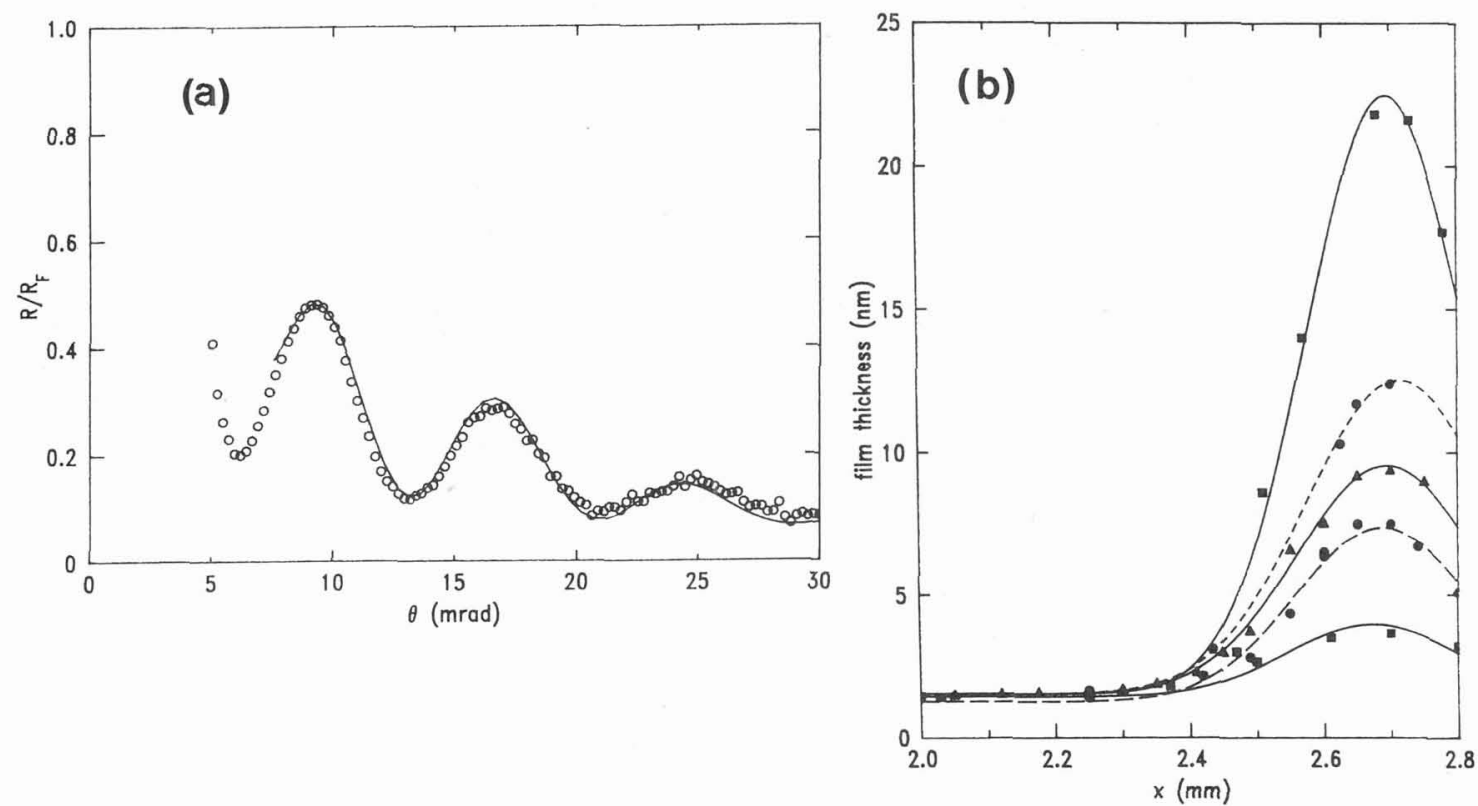

Fig. 14. (a) Reflectivity curve recorded at the edge of a droplet.

(b) Vanishing of the central bump.

A microscopic profile of the droplet can be obtained from a series of measures of the polymer thickness extracted from the reflectivity curves, at different positions on the sample. For example, a reflectivity curve measured on the very edge of the spreading droplet is shown in Fig. 14a. The complete profiles of a droplet $\left(\mathrm{V}=3.35 \times 10^{-7} \mathrm{~cm}^{3}\right) \mathrm{at}$ different s.tages of spreading are shown in Fig. 14b. These profiles can be fitted with a Gaussian function, as well as for the other droplets that we studied $\left(\mathrm{V}=2.4 \times 10^{-7} \mathrm{~cm}^{3}\right.$ and $3.4 \times 10^{-6} \mathrm{~cm}^{3}$, ). A Lorentzian shape has been predicted but this clearly does not agree with the experimental data. A striking feature to be noted is that the width of the Gaussian remains relatively constant during the spreading (Fig. 14b) 


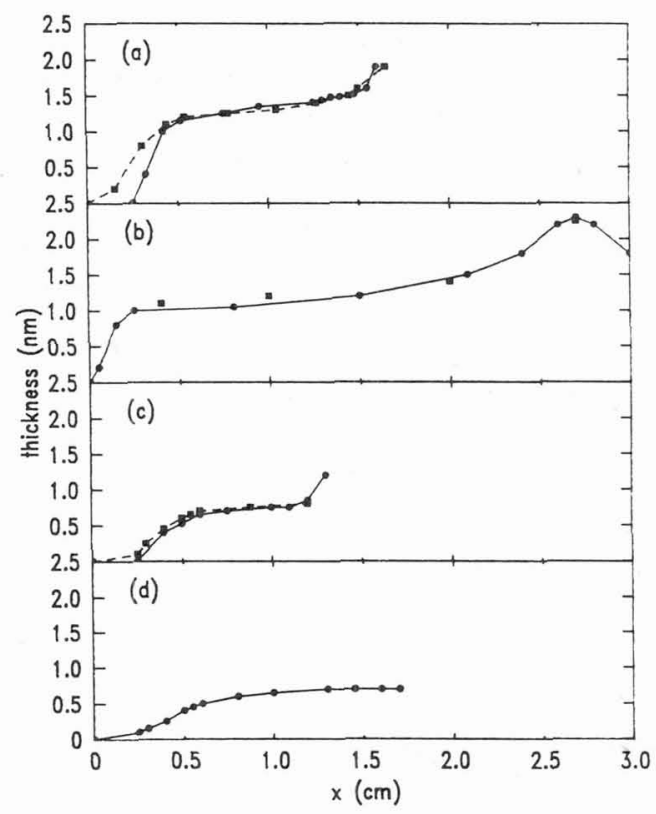

Fig. 15 : (a) The "tongue" on bare silicon, (b) The "straggler" (c) The tongue on a silanated wafer. (d) The "pancake" (silanated wafer).

Here, and in all the cases that were studied, the Gaussian part of the droplet is surrounded by a long, almost flat "tongue" (fig 15 a). Since the majority of the Gaussian part is contained within a $8 \mathrm{~mm}$ diameter, and the tongue extends over an area of about $10 \mathrm{~cm}^{2}$, almost nine tenths of the volume of the polymer is contained in the tongue. Moreover, the tongue develops very quickly, and only a slight shift of the contact line could be detected at the beginning of the $\mathrm{X}$-ray experiments. A first characteristic time of the spreading process is thus $\$ 1$ day.

In all of these experiments, the roughness of the substrate (bare silicon) was $4.7 \AA$, and that of the polymer film was $3 \AA$. The values of the density and the thickness over the majority of the tongue are then in good agreement with a single layer coverage of the substrate. Note that the measured thickness is larger than the molecular lateral dimension providing indirect information about the conformation of the chains.

The width of the droplet remaining essentially constant, the thickness of the maximum is a quantity of relevant physical significance. During the first weeks, variation in the height is compatible with a relaxation law whose time constant is on the order of 20 days. At these times, the edge of the tongue can be analyzed as a diffusion process. The entire set of data is in good agreement with a diffusion constant of $D=7 \pm 5 \times 10^{-7} \mathrm{~cm}^{2} / \mathrm{s}$. Thus, we observe mainly the vanishing of the Gaussian part into or through the tongue. 
Quite striking, as well, is an important slowing down of the spreading rate. The droplet shown on fig.14 did not vanish for the more than 200 days that it was followed after its deposition; a third time constant must be introduced, characteristic of the behaviour of what we shall call the "straggler" i.e. the remaining part of the central bump at long times (fig. 15.b).

The final stage of this system was presented in a previous paper[27]. The polymer film was described as non-homogeneous and monomolecular. It is worth noting that spreading does not stop; the tongue becomes more and more diffuse and the coherence of the liquid is broken. In this case, the theoretical picture of the so-called pancake is not relevant.

(b) Spreading of a droplet on a silanated silicon wafer

This system behaves much like the previous one; however two significative differences, must be underlined. In the case of spreading on silica, we were able to distinguish between three different steps with their characteristic times. Only the first two are observed with the silanated substrate and the final state has been shown to be of a very different nature[27]. In summation, the early stages of spreading appear to be quite similar, and we observe a Gaussian droplet surrounded by a flat tongue.

Let us remark, however, that the structure of the tongue is slightly different. It is a flat $\mathrm{film}$, only $7 \AA$ thick, with an abrupt edge (represented in $f i g .15 \mathrm{c}$ at $t=5$ days and $t=20$ days). This thickness is that of the polydimethylsiloxane chain with the methyl groups randomly oriented.

The most significant difference from the previous case is related to the later stages of spreading. The droplet $\left(\mathrm{V}=3.1 \times 10^{-7} \mathrm{~cm}^{3}\right)$ had completely spread within one month after its deposition, leaving a monomolecular, flat film on the substrate (fig. $15 \mathrm{~d}$ ) with well defined edges. This film was discussed in our previous study. It has been established that the theoretical pancake picture of de Gennes and Joanny is relevant; the spreading stops, and the equilibrium system is a finite thin flat liquid film. This state is in equilibrium, and the coherence of the liquid is not broken.

\section{(c) Results on the capillary rise}

Growth of the monomolecular film: The aim of these experiments was to get complementary information about the growth of the tongue at short times. Reflectivity curves were continuously recorded in order to investigate the growth of the film. The experimental data agree with a diffusion law; the diffusion constant $D$ has been determined $D-10^{-4} \pm 5 \times 10^{-5} \mathrm{~cm}^{2} \mathrm{~s}^{-1}$. This constant is consistent with our previous estimation since $\sim 1$ day was required to cover $\sim 10 \mathrm{~cm}^{2}$ of substrate.

Profile of the precursor film: The region just above the meniscus is represented in fig. 16 a few days after the beginning of the experiment. The data are well fitted with an $x^{-1}$ profile and the agreement is much better than with a $x^{-2}$ profile. Such an hyperbolic film is expected near the bulk surface of the liquid, the crossover length with the diffusive film being given by the meniscus velocity[32]. 


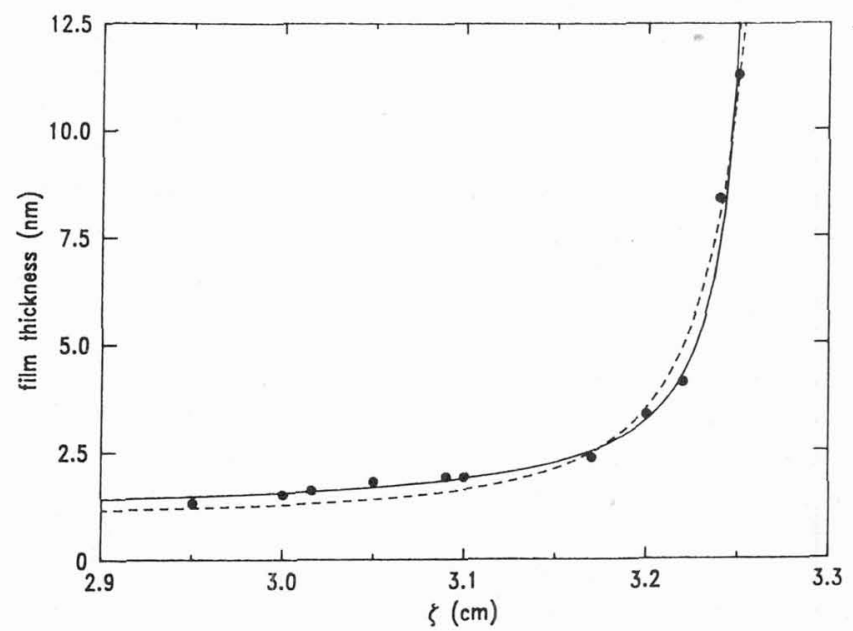

Fig. 16. Capillary rise : the precursor film (solid line, best $x^{-1}$ fit; dotted line, best $x^{-2}$ fit).

(3) Discussion

We shall discuss the experimental data using a rough model of surface flow which is only expected to exhibit the most important trends without completeness. An important point to be considered when discussing the data is to take advantage of the different experimental situations investigated, since their differences are indicative of the underlying molecular behaviour. The main problems to be discussed are: the growth of the tongue and its molecular structure, the profile of the central bump and, finally, the late stages.

(a) Surface flow

When the experiment involves a reservoir (e.g. the capillary rise), the rate of spreading is scaled by macroscopic phenomena and an hyperbolic "adiabatic" precursor film can be observed. With tiny droplets, no macroscopic reservoir is available, and the film is diffusive. It was established in ref. [33] that PDMS behaves as a liquid with a bulk viscosity down to $30 \AA$, but, obviously, in the case of monomolecular films, the flow process on the solid is more appropriatly considered as a surface diffusion process rather than as hydrodynamic. The problem is, then, to take into account the energy gained during spreading in the case of surface diffusion. Let us recall the main features of the model [34] . The molecular flow on the surface can be roughly described as follows: the transport of molecules is considered as a rate process, and an energy barrier is opposed to the hopping of molecules.

Since an energy $S$ is gained per unit area when covering the surface by a thin film of molecules, the barrier is distorted and non symmetric, and this leads to an acceleration of the process. Under these assumptions, the radius $R$ is such that $R=\sqrt{4 S / \eta_{e f f}} t^{1 / 2}$, where $\eta_{e f f}$ is an effective viscosity for the surface flow process. obviously, this law has to be carefully examined, but it displays satisfying trends: the 
velocity of the edge is proportional to the spreading parameter $S$ and inversely proportional to the effective viscosity and to the extension of the tongue. It is rather difficult to compare the processes which take place on bare and on silanated silicon wafers: $S$ is largex in the first case, but the friction is also larger since the chains are more strongly bonded to the substrate by hydrogen bonds. Therefore, no general conclusion can be drawn. Experimentally, the second effect appears to be the most important when comparing spreading on bare and on silanated silicon.

When the droplet is almost flat, the molecular flow has to relax toward a true diffusion process. The diffusion constant has been determined, and we have found $D=7 \pm 510^{-7} \mathrm{~cm}^{2} / \mathrm{s}$. The ratio of the diffusion constant for the surface energy driven process to that for the true diffusion process can be estimated, and we find 100 or more, which is consistent with the experimental data.

(b) The substrate-film interactions

From a general point of view, the substrate-liquid interactions are described using a disjoining pressure[35] which, in this case, is expected to have a long range molecular component due to dispersive forces and a structural component including the effects of hydrogen bonds. In the case of silanated wafers, the substrate is covered by a $20 \AA$ thick, dense hydrocarbon layer, and the interactions are mainly due to Van der Waals forces. Conversely, hydrogen bonds will play a major role for very thin films of PDMS on bare silicon surfaces. Shafrin and Zisman have studied polydimethylsiloxane from this point-of-view. They concluded that the methyl groups exposed by the silicone film are sufficiently closed-packed to shield the si-o linkages effectively from the wetting interface. Our data support this statement since a monomolecular coverage of the substrate was found. Moreover, the different thicknesses of the tongue measured on bare silicon $(15 \AA)$ and silanated silicon $(7 \AA)$ are indicative that the film may be constrained on bonding sites in the first case.

(c) The profile of the central bump

At thicknesses greater than $30 \AA$, PDMS can be considered as having its bulk hydrodynamic properties. An equation can be established within a lubrification approximation, and solutions have been proposed for a few cases[31]. The following mechanisin could explain why the droplet width remains almost constant: the liquid is sucked in the tongue while molecular forces oppose the thinning down of the central bump, whereas the spreading is hindered on the film itself due to its low critical surface tension.

(d) The "straggler"

The last point to be discussed is the retention of a thicker part (22A) at long times on bare silicon wafers. A general discussion cannot be carried out due to the lack of information about disjoining pressure, but the phenomena can be qualitatively understood as follows: The substrate is covered by two layers; the first layer of siloxane chains (15Å) exhibits methyl groups which considerably diminish the critical surface tension so that this surface is not very different from that of a silanated substrate. As we know, an equilibrium for spreading is reached on this surface, which is a molecular thick film $(7 \AA)$ in which cohesion prevails over diffusion. Since the two situations are close to each other, this equilibrium state is indicative of what can be expected on bare silicon and could explain the very long times required to completely spread the droplet. 
$\mathrm{X}$-ray reflectivity has been applied for the first time to the study of the phenomena of wetting. In summation, the spreading of a microscopic droplet involves at least two stages: the development of a "tongue" of molecular thickness is followed by a true surface diffusion process. Finally, the final stages have been shown to be largely dependent on the polymer-substrate interactions.

\section{Conclusion}

The techniques of $X$-ray reflectivity and surface diffraction have been applied to studies of many very different physical systems. These techniques using the optics of $\mathrm{X}$-rays have provided a means to measure physical properties that are non-accessible by other classical optical techniques.

Using a Langmuir-Blodgett multilayer film, we have shown how a detailed analysis of the complex interference pattern interfaces puts into evidence the role played by the reflected beams at the external interfaces compared to that of beams diffracted by the layers. This study leads to solutions to the more general problem of the phase determination in thin films.

Our study of a particularly simple monolayer system demonstrated the utility of

being able to separate the purely structural information from a measurement of the interfacial roughness. The 11 quid condensed to solid phase transition in behenic acid is characterized by the appearance of a strong bending rigidity that dramatically decreases the roughness of the monolayer. X-ray reflectivity is, to our knowledge, the only technique capable of measuring this effect.

Most original is the use of $X$-rays to study the macroscopic spreading of microscopically thin films. The wetting of polymers on solid surfaces have thus been studied. We have shown that the majority of the volume of a spreading micro-droplet is contained in a monomolecular thin film while the center has a Gaussian profile. Different final stages of the wetting have been identified for different substrate/polymer interactions. The geometry of the pure capillary rise has yielded a means of measuring the diffusion constant in the film. The use of X-ray reflectivity is completely free of the ambiguities inherent to an ellipsometric measurement of such ultra-thin films.

Finally, we have been able to perform these experiments using a conventional $X$-ray tube without having to resort to the high flux of a synchrotron source. This demonstration, which is of great practical importance, is due to the ultimate limitation being the intrinsic diffuse scattering from the sample. Thus, the proper use of a conventional source can equal the performance of a synchrotron for these types of studies, at a much greater convience.

\section{Acknowledgements}

We are indebted to P.G. de Gennes, F. Brochard and J. Meunier for their interesting and useful suggestions.

We wish to thank C. Blot and D. Luzet for their extremely competent technical asistance in the construction of our 4-circle diffractometer.

We are also infinitely grateful to A. Braslau for the many stimulating discussions as well as for carefully reading the text and to $J$. Ergotte for skillfully deciphering the hand written manuscript. 


\section{REFERENCES}

11/ Parrat, L.G., Phys. Rev. 95 (1954) 359

Croce, P. and Nevot, L., Revue Phys. Appl. 11 (1976) 113

12/ Marra, W.C., Eisenberger, P. and Cho, A.V., J. App1. Phys. 50 (1979) 6927.

13/ Brune1, M., Acta Cryst. A42 (1986) 304.

14/ Seul, M., Eisenberger, P. and Mc Connel, H.M., Proc. at. Sci. USA 80 (1983) 5795.

15/ Bohr, J., Feidenhansl, R., Nielsen, M., Toney, M., Johnson, R.L., Robinson, I.K., Phys. Rev. Lett. 4 (1985) 1275.

16/ Brunel, M. and de Bergevin, F., Acta Cryst. A42 (1986) 299.

17/ Bosio, L. and Oumezine M., J. Chem. Phys. $\underline{80}$ (1984) 954.

/8/ Rieutord F., Benattar, J.J. and Bosio, L., J. Phys. (Paris) 47 (1986) 1249.

19/ Abeles, F., Ann. Phys. (Paris) 5 (1950) 596.

Born, M. and Wolf, E., Principles of Optics, 6th edition (Pergamon Press, London) $1984,51$.

/10/ Rieutord, F., Benattar, J.J., Bosio, L., Robin, P., Blot, C. and de Kouchkovsky, R., J. Phys. (Paris), 48 (1987) 679.

/11/ Rieutord, F., Benattar, J.J., Rivoira, R., Lepêtre, Y., Blot, C., Luzet, D., to be published in Acta Cryst. (Section A).

/12/ Bouhet, C., Ann. Phys. (Paris), 15 (1931) 5.

/13/ Rasing, Th., Shen, Y.R., Kim, M.W. and Grubb, S., Phys. Rev. Lett. 55 (1985) 2903.

/14/ Kjaer, K., Als-Nielsen, J., Helm, C.A., Laxhuber, L.A. and Möhwald, H., Phys. Rev. Lett., 58 (1987) 2224 .

115/ Dutta, P., Peng, J.B., Lin, B., Ketterson, B., Prakash, M., Georgeopoulos, P. and Ehrlich, S., Phys. Rev. Lett., $\underline{58}$ (1985) 2228.

/16/ Bosio, L., Benattar, J.J. and Rieutord, F., Rev. Phys. App1. (Paris), 22 (1987) 775

/17/ Daillant J., Bosio, I., Benattar, J.J. and Meunier, J., Eurphys. Lett. $\underline{8}$ (5) (1989) 453-458.

18/ Beaglehole, D., Phys. Rev. Lett., $\underline{58}$ (1987) 1434.

119/ Earmshaw, J.C., Mc Givern, R.C. and Winch, P.J., J. Phys . (Paris) 49 (1988) 1271.

/20/ Braslau, A., Deutsch, M., Pershan, P.S., Weiss, A.H., Als-Nielsen, J. and Bohr, J., Phys. Rev. Lett., 54 (1985) 114

/21/ Braslau, A., Pershan, P.S., Swislow, G., Ocko, B.M. and Als-Nie1sen, J., Phys. Rev. A, $\underline{38}$ (1988) 2457.

122/ Cadenhead, D.A., Muller-Landau, F. and Kellner, B.M.J., in ordering in Two Dimensions, edited by Sinha (North Holland, Amsterdam) 1980, 73

/23/ Fischer, E, and Sackmann, E., J. Colloid. Interf, Sci, 112 (1986) 1.

/24/ Meunier, J., J. Phys. (Paris) 48 (1987) 1819.

/25/ Vig, J.R. in "Realise on clean surface technology" Vo. I, K.L. Mittal Ed. Plenum press, New York p.1, (1987).

/26/ Léger, I., Erman, M., Guinet-Picard, A.M., Ausséré, D., Strazielle, C., Benattar, J.J., Rieutord, F., Daillant, J., Bosio, L., Revue Phys. App1. 23 (1988) 1047.

/27/ Daillant, J., Benattar, J.J., Bosio, L. and Léger, L., Europhys. Lett. 6 (1988) 431.

/28/ de Gennes, P.G., Rev. Mod. Phys., 57 (1985) 827

Joanny, J.F., Thésis Université Paris (1985) unpublished.

/29/ Gun, J. and Sagiv, J., J. Coll, Int. Sci. 112 (1986) 457.

/30/ Tidswe11, I.N., Ocko, B.M., Pershan, P.S., Wasserman, S.R., Whitesides, G.M., preprint.

/31/ de Gennes, P.G., C.R. Acad. Sci. Sec.III 298 (1984) 75.

/32/ Joanny, J.F. and de Gennes, P.G., J. Phys. (Paris) 47 (1986) 121. 
/33/ Horn, R.G. and Israelashvili, J.N., Macromolecules 21 (1988) 2836.

/34/ Daillant, J., Benattar, J.J. and Léger, L., Submitted to Phys. Rev. A.

/35/ Derjaguin, B.V., Churaev, N.V. and Muller, V.M., Surface force (consultants Bureau, New York) 25 (1987). 